\title{
GAES - Guia de Acesso ao Ensino Superior
}

Fábio Oliveira de Sousa, ESBAM, fabio.olisousa@gmail.com

Márcio Aurélio dos Santos Alencar, UFAM, marcio.alencar@icomp.ufam.edu.br

Marcelo Batista de Souza, NEaD/UFRR, marcelo.souza@ufrr.br

José Francisco de Magalhães Netto, UFAM, jnetto@icomp.ufam.edu.br

\begin{abstract}
Resumo. Muitas pessoas tem dificuldade em escolher e ingressar em um curso de graduação. O presente artigo descreve o GAES, um aplicativo móvel desenvolvido na plataforma Android, auxiliado por um Sistema MultiAgente, com o intuito de ajudar futuros ingressantes do ensino superior na escolha de sua instituição de ensino e curso de graduação de acordo com seu perfil, suas preferências elou condições financeiras.
\end{abstract}

Palavras Chaves: Aplicações Móveis, Android, Sistemas Multiagente.

\section{GAES - Guide Access to Higher Education}

\begin{abstract}
Many people have difficulty choosing an undergraduate degree. This article describes the GAES, a mobile application developed on the Android platform, aided by a multi-agent system, in order to help future freshmen of higher education in their choice of institution and degree course according to your profile, your preferences and/ or financial condition.
\end{abstract}

Keywords: Mobile Applications, Android, Multiagent Systems.

\section{Introdução}

Até recentemente o fato de ter concluído o ensino médio era o suficiente para garantir um emprego bom e estável, porém atualmente muita coisa mudou e quanto mais qualificada a pessoa for, maiores serão suas possibilidades de encontrar um emprego que lhe assegure uma boa situação financeira. Tendo em vista que o mercado de trabalho tem exigido cada vez mais das pessoas que procuram por novas oportunidades, é imprescindível que elas se especializem em ao menos uma das três áreas existentes de ensino: Biológicas, Exatas ou Humanas, para ampliar suas chances de ser selecionado em uma entrevista de emprego. Isso mostra o quanto é importante o ensino superior no mercado de trabalho atual, pois um profissional formado, principalmente na área de atuação que mais se identifica, tem muito mais chances de alcançar o sucesso profissional.

As pessoas têm enfrentado grandes dificuldades em conseguir um espaço no mercado de trabalho, conforme os dados da Pesquisa Mensal de Emprego (PME), publicados pelo Instituto Brasileiro de Geografia e Estatística (IBGE), que apresenta um aumento de 6\% na taxa de desempregos no Brasil no mês de junho de 2013, o que resultou na maior taxa de desemprego desde abril de 2012 quando também atingiu 6\%, além disso, a pesquisa informa que junho é o sexto mês seguido que o desemprego aumenta (IBGE, 2013). 
Devido ao aumento do desemprego, as pessoas tendem a buscar por uma formação superior para se especializar e tentar garantir uma vaga no mercado, porém encontram alguns obstáculos no caminho até a faculdade, um deles é que as informações dos cursos disponibilizados pelas instituições de ensino geralmente são incompletas e de difícil acesso, outro obstáculo e talvez mais significativo é o fato de que muitas pessoas não sabem realmente que profissão seguir, segundo a pesquisa divulgada no jornal o Estado de São Paulo e realizada pelo Portal Educacional com 2 mil jovens em 22 de agosto de 2011, a indecisão no momento de escolher um curso é um problema enfrentado por cerca de $54 \%$ dos estudantes concluintes do Ensino Médio, e essa mesma pesquisa mostra que, quando as instituições abrem as inscrições para os processos seletivos, muitos alunos ainda não tem ideia de qual carreira seguir. (Balmant, 2011)

É importante saber escolher bem o curso de graduação e desenvolver todas as competências necessárias que o mercado de trabalho exige, e esse é um passo muito importante, pois estão definindo um caminho a ser seguido por toda a vida. Além do curso é preciso definir também em qual faculdade estudar, pois há diversas possibilidades, e atualmente somente na cidade de Manaus, existem mais de 20 faculdades particulares, sendo que a maioria tem localizações distantes umas da outras, o que torna inviável à visita a cada uma delas por parte dos candidatos interessados em ingressar em um curso superior, dada a distância e o tempo que seria utilizado para isso. Em busca de informações que facilitem seu ingresso em uma faculdade, as pessoas tendem a procurar por divulgações realizadas pelas instituições de ensino nos meios de comunicação (Jornal, Tv, Rádio, revista e etc.) ou até mesmo em seus sites oficiais, contudo as informações determinantes para o ingresso, principalmente referentes aos valores dos cursos, muitas vezes não são disponibilizadas e obtidas somente se o interessado comparecer ao endereço físico da instituição.

O presente artigo propõe como solução para esse problema a criação do aplicativo móvel GAES (Guia de Acesso ao Ensino Superior), baseado em Sistema MultiAgente (SMA), usando o framework JADE (Java Agent DEvelopment Framework). O aplicativo é dotado de funcionalidades que facilitam a escolha de um curso de nível superior em faculdades particulares brasileiras, disponibilizando todas as informações necessárias para o ingresso do novo aluno.

\section{Trabalhos Correlatos}

Nos últimos anos é crescente o número de dispositivos portáteis, que em conjunto com a alta necessidade de acesso em movimento à diversas informações, tem modelado o caminho para uma nova tecnologia que combina a telefonia móvel com o mundo da $W e b$. Essa tendência que já virou realidade só tem aumentado a cada dia, pois esses dispositivos além de serem mais fáceis de transportar, ainda contam com diversos recursos multimídia que atraem cada vez mais adeptos. Esse crescimento impulsionou o mercado de aplicativos para dispositivos móveis popularmente chamados de 'apps'. Nesse contexto os trabalhos citados nessa seção serviram de base para o estudo e desenvolvimento do aplicativo proposto.

Santos e Almeida (2012) desenvolveram um aplicativo em android para facilitar o ingresso de novos estudantes na Universidade Tecnológica Federal do Paraná UTFPR, apresentando em qualquer dispositivo móvel munido com o sistema operacional android o catálogo de cursos disponibilizados pela universidade, permitindo a visualização de todas as informações e características dos cursos oferecidos em todos 
os campus da instituição. As informações disponibilizadas no aplicativo são extraídas do site da universidade através de um recurso disponível para a plataforma de programação Java conhecido como JSoup (Java HTML Parser) que é uma biblioteca da linguagem que permite a captura de conteúdo HTML. A versão do Android utilizada para a implementação do sistema foi a 2.3.3, por se tratar da versão mais utilizada no ano de seu desenvolvimento.

Puniani (2011) elaborou o protótipo de um aplicativo intitulado School Mate, para ajudar estudantes a encontrar docentes dentro de uma instituição de ensino, pelo fato de que todos os alunos precisam estar sempre em contato com seus professores, seja para tirar dúvidas sobre os cursos em andamento, pedir orientação em trabalhos ou qualquer tipo de dificuldade que surgir no ambiente acadêmico, tendo em vista que nas grandes universidades que contém várias unidades, é muito difícil principalmente para novos alunos localizar o departamento em que o professor está em determinado tempo, o aplicativo fornece ao estudante três opções de busca: departamento, nome ou sobrenome, e ao final da busca o aplicativo traz a localização do educador mostrada em um mapa. O Sistema foi desenvolvido utilizando a plataforma android e conta com uma aplicação web desenvolvida em JSP (Java Server Pages) para fornecer aos discentes, informações referentes a horário de expediente, departamento, contatos (e-mail e telefone) e localização dos educadores.

O trabalho de Santi et al (2010) relata a importância da AOP (Agent-Oriented Programming) ou Programação Orientada a Agentes, aplicada a projetos que usam dispositivos móveis como Android e iPhone. Para auxiliar nas pesquisas, eles desenvolveram o framework Jaca-Android, que usa a plataforma Google Android, contribuindo com a programação de Agentes Inteligentes. Podemos citar também a arquitetura denominada MOA (Micro-agents on Android), desenvolvida por Frantz et al (2011), que faz a integração de Micro-Agentes com a plataforma Android, além de permitir a construção de aplicações hibridas. Já o trabalho de Lawrence e Sankaranarayanan (2012) apresentou uma arquitetura interligando um aplicativo móvel com agentes inteligentes para ajudar usuários na reserva de hotéis.

\section{Tecnologias empregadas}

No desenvolvido do GAES foi utilizando a plataforma open-source IDE Eclipse, o SDK (Software Development Kit) com o plugin ADT (Android Development Tools) que permite criar aplicações para o sistema operacional Android. A aplicação web foi desenvolvida e testada usando o software Xampp, composto pelo servidor web Apache, o banco de dados MySql e a linguagem de programação PHP. As páginas em HTML e PHP foram editadas usando o software Adobe DreamWeaver. Para localização da faculdade, foi utilizado o serviço de GPS do Android juntamente com o serviço de mapas do Google.

É notável o crescimento de pesquisas utilizando o GPS (Global Positioning System) dos dispositivos. Como uso dessa tecnologia temos o StoreFinder, desenvolvido por Debastiani et al (2011), um aplicativo móvel que usa GPS e TV Digital, para verificar a localização atual do usuário e mostra um mapa com a rota desta posição até a empresa cujo comercial está sendo recebido no dispositivo móvel. A aplicação MobiLE, desenvolvida por Silva et al (2011) que coleta a localização geográfica do estudante e 
com ajuda dos três agentes inteligentes, realizam a identificação do perfil do estudante de Ead.

Foi implementado um Sistema Multiagente (SMA), que é uma sociedade de agentes que trabalham em conjunto sobre um ambiente na busca pela resolução de um problema [Wooldridge, 2009]. Para desenvolver, implementar e testar os agentes inteligentes, foi utilizado o framework JADE, um software livre que usa a linguagem de programação Java e especificações da FIPA (Foundation for Intelligent Physical Agents) [Bellifemine et al, 2007].

\section{Arquitetura}

Na Figura 1, pode-se observar a arquitetura do GAES, composta pelo administrador do Sistema, o Usuário do aplicativo móvel e os agentes inteligentes.

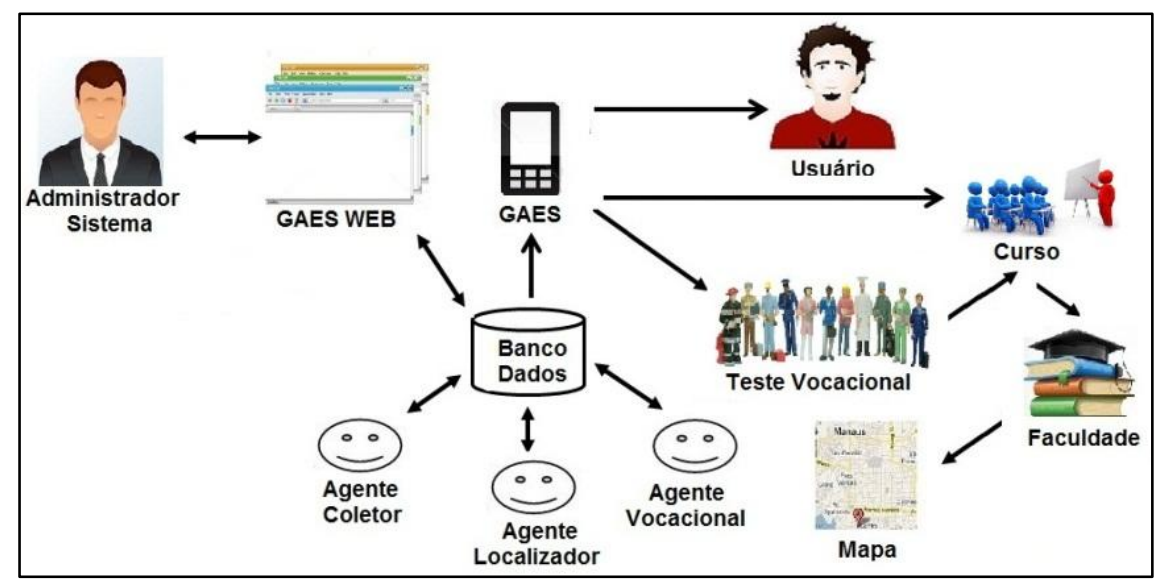

Figura 1 - Arquitetura do GAES

- Administrador do Sistema - responsável por cadastrar e manter atualizado os dados das faculdades particulares no GAES Web.

- GAES Web - Sistema Web desenvolvido em PHP que utiliza o banco de dados MySQL.

- Usuário - Utiliza o aplicativo em seu smartphone ou tablet.

- GAES - Aplicativo na plataforma Android.

- Banco de Dados - As informações das faculdades e dos usuários são armazenadas no banco de dados MySql, que é utilizado pela versão web e Android.

- Agente Coletor - responsável por apresentar informações detalhadas da faculdade e do curso selecionado pelo usuário.

- Agente Localizador - responsável por apresentar a localização da faculdade usando o googlemaps.

- Agente Vocacional - responsável por coletar consultar informações na base de dados, sugerindo vocações aos usuários, assim como coletar informações sobre os testes vocacionais realizados.

- Teste Vocacional - Ferramenta que sugere vocações ao usuário de acordo com seu perfil. 
- Curso - Curso superior escolhido ou sugerido pelo GAES.

- Faculdade - São presentadas no GAES informações detalhadas sobre a faculdade.

- Mapa - É mostrado no GAES, por meio da ferramenta GoogleMaps, o caminho que o usuário irá percorrer até chegar a faculdade.

\section{Experimentos}

Existem diversas pesquisas que usam SMA para auxiliar a educação, como os trabalhos de Reategui et al (2008) e Alencar e Netto (2012), que utilizaram essa tecnologia aplicada a um Ambiente Virtual de Aprendizagem (AVA) para facilitar a colaboração e aprendizado de estudantes. Já em nosso trabalho, avaliamos o aplicativo, composto por um SMA, representado pelos agentes (Coletor, Localizador e Vocacional).

Os testes utilizaram uma base de dados apenas com informações de faculdades da cidade de Manaus, onde simulamos que 06 (seis) usuários de perfis diferentes, utilizando as diversas funcionalidades do GAES. O aplicativo GAES, em seu menu principal apresenta uma gama de opções, para facilitar a busca por um curso de graduação, conforme Figura 2.

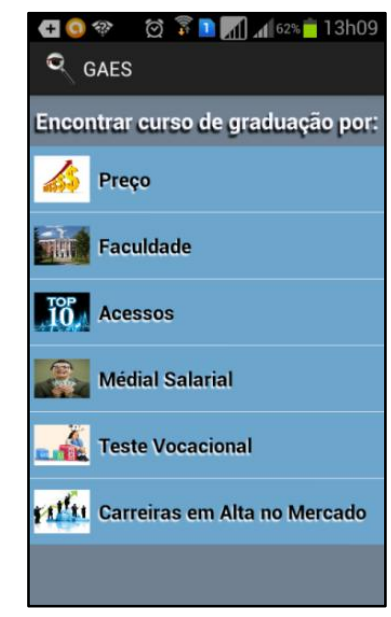

Figura 2 - Tela principal

O usuário 1 escolheu a opção "preço" no menu e na tela de busca escolheu o Estado, a Cidade, o curso que pretende estudar, a modalidade, que pode ser presencial ou à distância e o turno: matutino, vespertino ou noturno, conforme Figura 3a.

Com base nas informações repassadas pelo usuário, o agente coletor realiza uma pesquisa no banco de dados, redirecionando o resultado ao usuário, onde são mostradas em forma de lista todas as instituições que disponibilizam o curso com os critérios escolhidos pelo usuário, inicialmente essa lista é organizada por um ranking de preço, ou seja, uma lista ordenada de forma crescente conforme o menor preço do curso escolhido.

$\mathrm{Na}$ tela de "Busca Concluída", é mostrado o curso, modalidade e turno selecionado. Se preferirmos temos a opção de "organizar por", que reorganiza a lista por: preço, bolsa universidade, pró-uni ou média Enade/Mec (Exame Nacional de 
Desempenho de Estudantes do Ministério da Educação), podendo essas informações serem reorganizadas de forma crescente ou decrescente, conforme o usuário desejar.

A lista apresentada ao usuário mostra no lado esquerdo os logotipos das instituições e a data de próximo vestibular; no meio da lista estão, os nomes das instituições de ensino e seus respectivos endereços; e ao lado direito da lista estão localizados inicialmente os preços dos cursos (em destaque), a quantidade de bolsas de estudos disponibilizadas para esses cursos pelo programa Bolsa Universidade (programa de bolsas de estudo da prefeitura da cidade), a quantidade de bolsas de estudos disponibilizadas pelo programa Pró-Uni (Programa Universidade Para Todos) e as notas dos cursos no exame Enade/Mec, conforme Figura 3b.

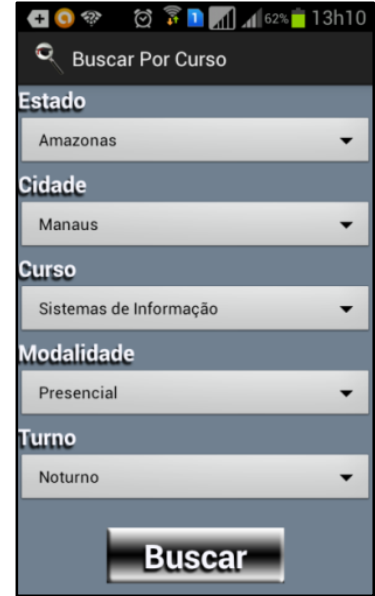

Figura 3 - a) Tela de busca

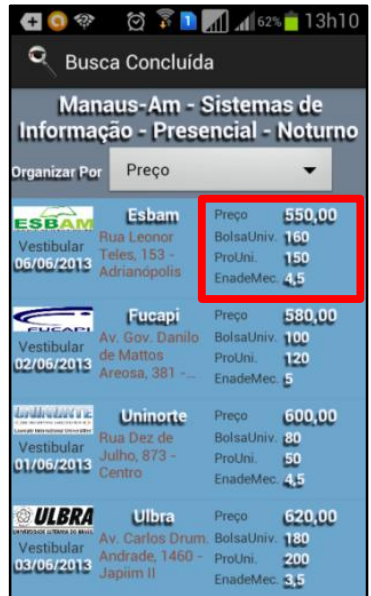

b) Resultado da busca por preço

Ao clicar no logotipo de qualquer uma das instituições apresentadas na lista, o agente coletor redireciona o resultado da busca para a tela "Faculdade selecionada", como podemos ver na Figura 4, trazendo informações que considera-se essencial para que o usuário possa escolher seu curso e a instituição em que irá estudar, são elas: duração total do curso (períodos), quantidade de horas complementares exigidas para a conclusão do curso, grade curricular, quantidade de bolsas para o programa Bolsa Universidade e ProUni de acordo com a porcentagem da bolsa, a área de conhecimento, a formação profissional, as áreas de atuação do curso escolhido, além da integração com as redes sociais, por meio dos ícones (twitter e facebook).
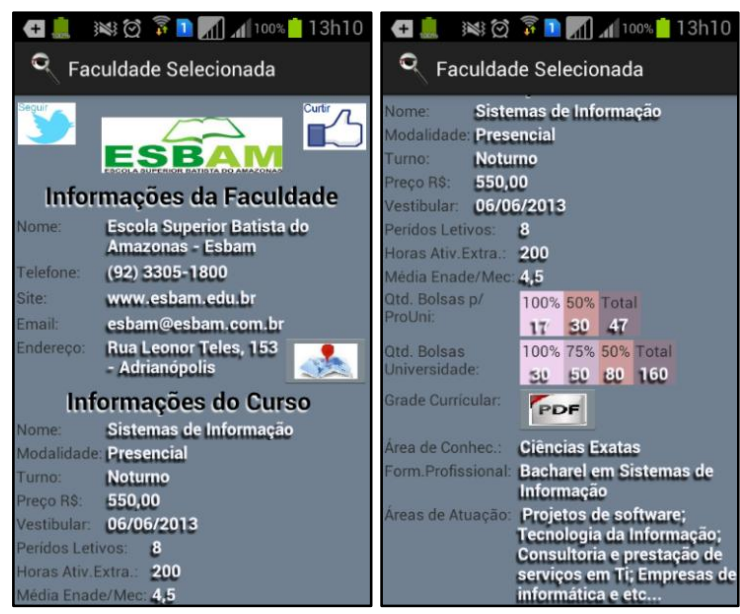

Figura 4 - Informações da faculdade e do curso selecionado 
Além dessas informações, o usuário pode visualizar o endereço da faculdade em um mapa do Google Maps, para isso o agente localizador recebe informações sobre a faculdade, gera o mapa e calcula a distância de seu ponto até a faculdade, conforme figura 5a. No mapa podemos visualizar a localização da faculdade juntamente com a localização do usuário, mostrando inclusive um cálculo de distância entre os dois pontos.

O usuário 2 escolheu a opção "faculdade" no menu principal, informou o estado, cidade e faculdade em que deseja estudar, então o agente coletor realizou consulta na base de dados e retornou as informações de todos os cursos disponíveis na instituição escolhida conforme Figura 5b.

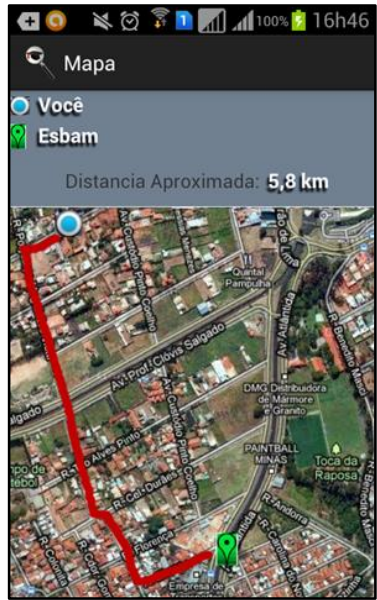

Figura 5 - a) Tela de localização da faculdade

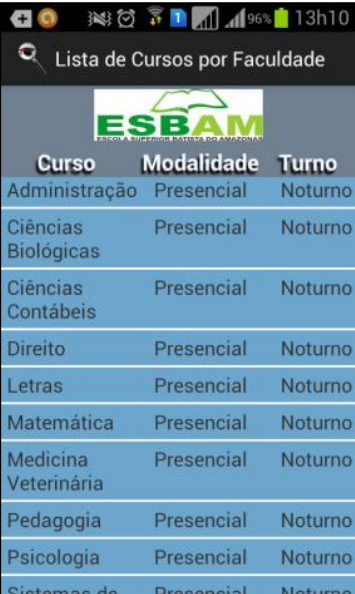

b) Cursos por faculdade

O usuário 3 escolheu a opção "média salarial", então o agente coletor pesquisou na base de dados os 10 (dez) cursos de graduação mais bem remuneradas, com sua respectiva média salarial do mercado de trabalho, conforme figura $6 \mathrm{a}$, oferecendo assim mais um parâmetro para os usuários decidirem que curso pretendem cursar. $\mathrm{O}$ usuário 4 escolheu a opção "acessos", então o agente coletor verificou na base de dados todos os acessos e interesses dos usuários por um determinado curso e retornou os cursos mais acessados com a quantidade de acesso, conforme Figura $6 \mathrm{~b}$.

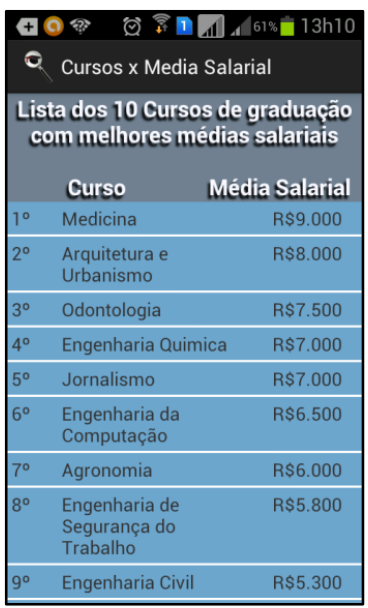

Figura 6 - a) Cursos e média salarial

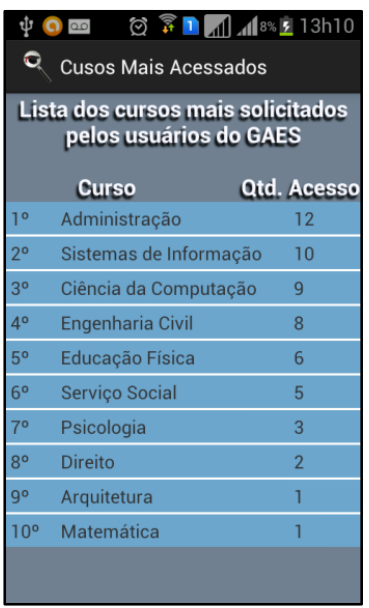

b) Cursos por acesso

O usuário 5, simbolizando as pessoas que não tem a mínima ideia de que 
carreira seguir, escolheu no menu o "Teste Vocacional", onde respondeu 10 (dez) questões, como podemos ver na Figura 7a, com isso o agente vocacional verificou seu perfil e que área de atuação pode seguir, utilizando para isso a base de dados, recomendando assim uma lista de cursos de acordo com as respostas coletadas, visto na Figura $7 b$.

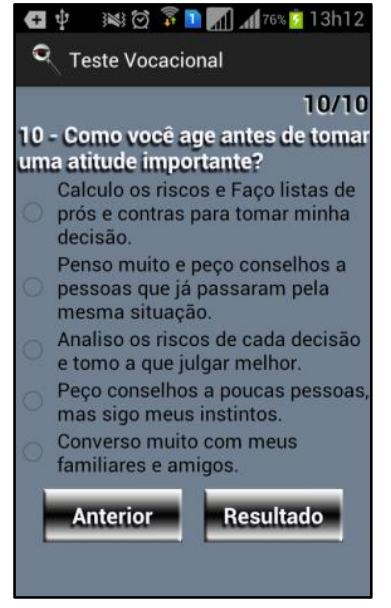

Figura 7 - a) Questionário teste vocacional

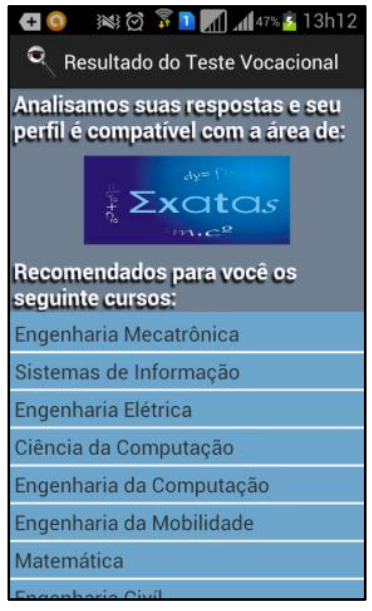

b) Resultado teste vocacional

O usuário 6 resolveu escolher "Carreiras em Alta", com isso acionou o agente coletor, que fez pesquisas na base de dados e mostrou uma lista com as carreiras em alta no mercado e com grandes perspectivas de crescimento conforme figura 8 . A lista inclui a profissão e o curso que o usuário pode estudar para exercer a profissão.

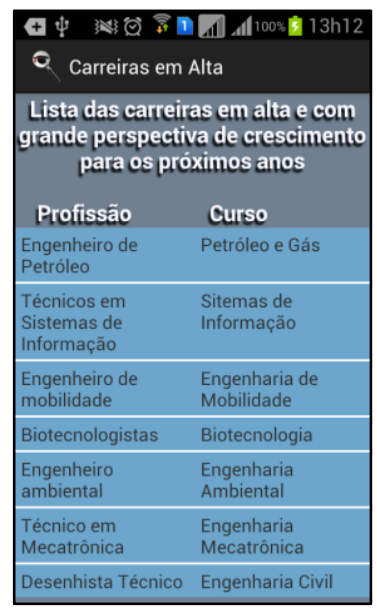

Figura 8 - Tela carreiras em alta

\section{Conclusões e Trabalhos Futuros}

Este artigo apresentou o GAES, um aplicativo móvel que auxilia pessoas na busca por cursos de graduação, apoiando a descoberta de interesses e vocações, apresentando opções de cursos conforme seu perfil ou suas próprias preferências, e fornecendo todas as informações necessárias para uma boa escolha.

Notou-se que aplicativos dessa categoria são de essencial importância para futuros alunos do ensino superior, tendo em vista que existem diversas instituições de ensino que oferecem os mais variados cursos e temos uma grande dificuldade em visitar 
cada uma delas para realizar pesquisas, além disso, outro fato importante é que muitas pessoas não sabem nem mesmo que carreira seguir, o que agrava a dificuldade de escolher um curso de graduação. Com base nesse contexto, acredita-se que o aplicativo GAES irá possibilitar uma melhor integração entre pessoa e faculdade, além de proporcionar um aumento na quantidade de alunos que ingressam semestralmente no ensino superior.

Como trabalhos futuros pretendemos melhorar os comportamentos dos agentes, além de criar novos agentes inteligentes que possibilitem a consulta por cursos de pósgraduação, e que apresentem também informações sobre as faculdades públicas. Além de implementar um sistema de recomendação para trabalhar em conjunto com o agente vocacional, com a intenção de melhorar a recomendação de cursos para os usuários de perfis semelhantes.

\section{Referências}

ALENCAR, M. A. S.; NETTO, J. F. M. (2012). Sistema Multiagente para Apoiar a Percepção e o Acompanhamento de Atividades em Ambientes Virtuais de Aprendizagem. In: XXIII SIMPÓSIO BRASILEIRO DE INFORMÁTICA NA EDUCAÇÃO, 2012, Rio de Janeiro, RJ. Anais do XXIII Simpósio Brasileiro de Informática na Educação, 2012.

BALMANT, O. Metade dos alunos do $3^{\circ}$ ano não sabe qual carreira seguir. Jornal o Estado de São Paulo. 22 ago 2011. Disponível em $<$ http://www.estadao.com.br/noticias/impresso,metade-dos-alunos-do-3-ano-nao-sabequal-carreira-seguir,761850,0.htm>. Acesso em 15 out. 2013.

BELLIFEMINE, F.; CAIRE, G.; GREENWOOD, D. Developing multi- agent systems with JADE. 1.ed. England: Editora Wiley, 2007, 300 p., ISBN: 9780470057476.

DEBASTIANI, D.; VIEIRA, A.; SILVEIRA, M.; MARQUES, F.; AGOSTINI, L. StoreFinder: Um aplicativo Android para TV Digital utilizando recursos de Mapas e GPS. In: Seminário de Pesquisa em Computação, Pelotas - RS, 2011.

FRANTZ, C.; NOWOSTAWSKI, M.; PURVIS, M. K. Micro-agents on Android: Interfacing Agents with Mobile Applications. In: 10TH INTERNATIONAL CONFERENCE ON ADVANCED AGENT TECHNOLOGY. AAMAS, 488-502, 2011.

IBGE - Instituto Brasileiro de Geografia e Estatística. Pesquisa Mensal de Empregos: Estimativas para o Mês de Junho de 2013 - Recife, Salvador, Belo Horizonte, Rio de Janeiro, São Paulo e Porto Alegre, 2013.

LAWRENCE, W. SANKARANARAYANAN, S. Smart Agent Learning based Hotel Search System- Android Environment. In: International Journal of Information Technology and Computer Science - IJITCS, ISSN: 2074-9015, 9-12, 2012.

PUNIANI, P. School Mate - Instructor Finder. California. San Diego: San Diego State University, 2011. 44p. Master of Science in Computer Science. 
REATEGUI, Eliseo; RIBEIRO, A. M.; BOFF, Elisa. Um Sistema Multiagente para Controle de um Assistente Pessoal Aplicado a um Ambiente Virtual de Aprendizagem. Revista Novas Tecnologias na Educação - RENOTE, v. 6, p. 7-17, 2008.

SANTOS, W. S; ALMEIDA, M. S. O. Produção de aplicativo de catálogo de cursos da UTFPR para o sistema Android. In: III SEMINÁRIO DE EXTENSÃO E INOVAÇÃO DA UTFPR - SEI, 2013.

SANTI, A.; GUIDI, M.; RICCI, A. JaCa-Android: An Agent-Based Platform for Building Smart Mobile Applications. In: INTERNATIONAL WORKSHOP ON LANGUAGES, METHODOLOGIES AND DEVELOPMENT TOOLS FOR MULTI-AGENT SYSTEMS - LADS 2010.

SILVA, L. C. N.; MENDES NETO, F. M.; JÁCOME JÚNIOR, L. MobiLE: Um Ambiente Multiagente de Aprendizagem Móvel para Apoiar a Recomendação Sensível ao Contexto de Objetos de Aprendizagem. In: XXII SIMPÓSIO BRASILEIRO DE INFORMÁTICA NA EDUCAÇÃO - SBIE, 2011, Aracaju, SE.

WOOLDRIDGE, M. J. An Introduction To Multiagent Systems. 2.ed. England: John Wiley \& Sons. 2009, 484 p, ISBN: 978-0470519462. 